\title{
La (des)contextualización del conocimiento geográfico en el manejo del riesgo de incendios forestales en Chile como un desafío para la gobernanza ${ }^{1}$
}

\section{The (de-)contextualization of geographical knowledge in forest fire risk management in Chile as a challenge for governance}

\author{
Michael Handke
}

\begin{abstract}
RESUMEN
La economía y la sociedad chilena se enfrentan a riesgos crecientes de incendios forestales. Predominan las soluciones de gestión de riesgos individuales en lugar de las colectivas. Las grandes empresas forestales reducen la probabilidad de que los incendios afecten a sus plantaciones con rutinas de gestión de orden jerárquico, compran pólizas de seguro para protegerse de pérdidas económicas desastrosas y diversifican y externalizan su riesgo en el mercado. Estos mecanismos no son disponibles para todos actores en las regiones forestales chilenas que experimentan los incendios principalmente como efectos externos negativos. El articulo evalúa las ventajas y desventajas de las formas jerárquicas y mercantiles de la gestión del riesgo y denuncia la necesidad de un enfoque geográfico más profundo en cuanto a la gobernanza. Así, se revela que el conocimiento geográfico detallado sobre los riesgos de incendios forestales se descontextualiza explícitamente en las prácticas actuales de gestión de riesgos en Chile. El conocimiento detallado de las causas y efectos de los incendios, se pierde en el proceso.
\end{abstract}

Palabras clave: Gestión del riesgo de incendios forestales, gobernanza del riesgo, incertidumbre, seguros, plantaciones forestales

\begin{abstract}
The Chilean economy and society are confronted with increasing risks from forest fires. Individual rather than collective risk management solutions predominate. Large forest companies reduce the probability that forest fires affect their tree plantations with management routines of hierarchical order. Additionally, they purchase insurance policies to protect themselves from disastrous economic losses. They diversify and externalize their risk. These mechanisms are not available to other stakeholders in Chilean forest regions who experience forest fires mainly as negative external effects. The paper assesses the strengths and weaknesses of interacting hierarchical and market governance forms
\end{abstract}

Agradecimientos: Esta investigación fue apoyada por el Heidelberg Center para América Latina y su programa de Magister en Gobernanza de Riesgos y Recursos financiado por el DAAD. Además, los recursos financieros de la Universidad de Heidelberg contribuyeron en gran medida al éxito del proyecto de investigación multianual. Agradezco a los numerosos estudiantes del máster que han contribuido a dar forma a los argumentos del trabajo a través de la recopilación de datos y de innumerables debates. Un agradecimiento especial a Tatiana Patiño por su incansable trabajo en contactar el sector asegurador chileno. 
of risk management and calls for a deeper geographical approach of risk governance. It reveals that detailed geographical knowledge on forest fire risks is explicitly decontextualized and even ignored in current risk management practices. Knowledge of the causes and effects of forest fire risks, that is important for a broader approach of risk governance, is lost in the process.

Keywords: Forest fire risk management, risk governance, uncertainty, insurance, plantation forest

En enero de 2017 Chile experimentó los peores incendios forestales de la historia reciente. Más de 120 incendios simultáneos en las regiones de O’Higgins, Maule y Biobio afectaron casi 467.000 hectáreas de bosque nativo y plantaciones forestales (CONAF, 2017). Los daños económicos de los incendios fueron significativos. Por ejemplo, Empresas CMPC S.A. -un holding multinacional de origen chileno, fabricante de papel y la segunda empresa forestal más grande del país, con una propiedad forestal de alrededor de 487.000 hectáreas- anunció que el área de plantaciones afectadas por los incendios alcanzó aproximadamente 19.000 hectáreas, lo que equivale a un daño económico de US\$73 millones. Posteriormente, la empresa informó que, a pesar de estar cubierta por pólizas de seguro, la compensación por daños se limitaba a US\$17 millones debido a los deducibles especificados en los contratos de seguro. Por último, CMPC aseguró que el suministro de madera para las operaciones de la planta no se vio afectado. El beneficio antes de impuestos del holding al cierre del ejercicio fue de US\$ 117 millones (CMPC, 2018: 141).

Declaraciones como estas, que se publican en los informes anuales de las grandes empresas forestales chilenas, demuestran claramente que desde el punto de vista económico es posible disociar la dimensión física del riesgo -es decir, la ocurrencia de incendios forestales en el espacio- de su dimensión económica -la pérdida de áreas forestales- y financiera -las advertencias sobre los beneficios. Por un lado, las estrategias individuales de las empresas para controlar el riesgo consisten en la aplicación de normas modernas de gestión de plantaciones forestales que incluyen, entre otras cosas, el mantenimiento de torres de vigilancia de incendios y el empleo de sus propios cuerpos de bomberos. Así, reducen las probabilidades de que se produzcan incendios y controlan la magnitud de las pérdidas con órdenes jerárquicas y determinadas rutinas (véase también Perrow, 1986). Para ello, coordinan procesos de toma de decisiones centralizados y descentralizados tanto en el espacio legal de la empresa como en el espacio físico en el que se ubican las unidades operativas. Por otro lado, las empresas aplican una solución de gestión de riesgos de mercado al comprar contratos de seguros que les permiten cubrir parte de las pérdidas económicas (Chichilinsky \& Heal, 1998). El seguro transforma el riesgo en un bien comercial. El asegurado paga una prima de seguro para compensar al asegurador por asumir el riesgo. En otras palabras, el asegurado renuncia a parte de sus beneficios y los convierte en certeza (Freeman \& Kunreuther, 1997). El mercado de seguros como instrumento de gestión de riesgos para reducir la vulnerabilidad económica de los actores sociales ha adquirido un creciente interés en Chile (Dean et al, 2003; Loewe et al, 2017).

Con sus memorias anuales, las grandes empresas forestales en Chile señalan un conocimiento específico y un lenguaje sofisticado sobre el valor del riesgo para los mercados financieros (Kalthoff, 2005). En este proceso, los incendios forestales individuales y el daño que causan se disocian del contexto geográfico real en el que se originan. Los riesgos se calculan con la ayuda de modelos técnicos de riesgo, y se cuantifican para ser traducidos y comunicados hacia el mercado 
en donde pueden ser diversificados. La cuantificación restringe el conocimiento sobre el riesgo (Beck, 1998; Luhmann, 2006). En el curso de la cuantificación del riesgo ya no es fácil comprender cómo se producen los incendios forestales y qué consecuencias sociales adicionales puedan tener. Una sociedad que se basa únicamente en prácticas de gestión del riesgo económico pierde su capacidad de responder adecuadamente a las diferentes causas y consecuencias del riesgo, y en particular, a su evolución (Rosa et al., 2014).

Las estrategias y prácticas para enfrentar el riesgo de incendios forestales en Chile son un objeto apropiado de investigación conceptual y empírica (Úbeda \& Sarricolea, 2016). Los devastadores incendios forestales que se registraron en Chile a comienzos del 2017 han aumentado la sensibilidad general de la sociedad a este tipo de riesgo. Revelaron la vulnerabilidad de varios miembros de la sociedad y evidenciaron su impotencia para tomar decisiones reales sobre los riesgos. Mientras que la gestión del riesgo de las empresas forestales y de las compañías de seguros se ejecuta principalmente en privado, donde los balances anuales indican que los incendios forestales son económicamente controlables, otras partes de la sociedad que también se ven afectadas están luchando por hacer frente a las consecuencias de estos. Los incendios forestales también amenazan los medios de vida de parte de la población en las regiones forestales chilenas. Esto no solo plantea la cuestión de la forma adecuada en que la sociedad debe tratar el riesgo. Al mismo tiempo, surge un interés creciente de abrir la caja negra de los sistemas internos de gestión de riesgos de las empresas para detectar todo el espectro de efectos externos negativos (Van Dam, 2006; Bottaro, 2018). La adecuación de las formas jerárquicas y mercantiles de la gestión del riesgo para mejorar la prevención del riesgo de incendios forestales en la sociedad chilena o, en términos generales, para aumentar la resiliencia de la sociedad al riesgo -que es la preocupación de la perspectiva más amplia de la gobernanza del riesgo- sigue siendo objeto de debate, lo que motiva el siguiente análisis.

La gestión del riesgo y la gobernanza del riesgo no son lo mismo. Las diferencias radican no solo en la perspectiva de los actores involucrados (manejo individual de riesgos versus manejo colectivo) o en el horizonte temporal que sustenta sus prácticas (orientación a corto plazo versus orientación a largo plazo), sino sobre todo en la producción y uso de conocimientos avanzados sobre riesgos: "Los riesgos surgen en el conocimiento, y por lo tanto en el conocimiento pueden ser reducidos, ampliados o simplemente eliminados de la conciencia" (Beck, 1998: 84). Una perspectiva de conocimiento del riesgo promete clarificar las diferencias entre la gestión y la gobernanza, y también explica las interrelaciones entre las diferentes prácticas de gestión del riesgo. Ello conduce a la siguiente pregunta de investigación: ¿Cómo pueden las formas jerárquicas y mercantiles de la gestión del riesgo complementarse en el proceso de la gobernanza del riesgo? Al revelar las ventajas y desventajas de la interacción entre las formas jerárquicas y de mercado y centrarse en los desafíos epistemológicos del riesgo de incendios forestales en Chile, el artículo contribuye a una mejor comprensión de los beneficios sociales de la organización de una gobernanza del riesgo explícitamente regional y colectiva, a pesar de que aún no existe en el país.

El artículo se divide en cuatro partes. Tras esta introducción, en la sección uno se explica y justifica la relevancia del problema de investigación en relación con los desafíos que plantean las diferentes perspectivas del conocimiento para afrontar los riesgos. Se distingue explícitamente entre gestión del riesgo y una perspectiva de gobernanza del riesgo. La sección dos analiza las 
diferentes características de los incendios forestales en Chile. Se argumenta que las relaciones entre las causas y los efectos de los incendios forestales son epistemológicamente complejas, ambiguas e inciertas. La sección deja claro que para entender la complejidad, ambigüedad e incertidumbre en relación con los incendios forestales se requiere una visión espacio-temporal deliberada. Estos resultados se contrastan en la sección tres con un análisis de las prácticas de las empresas forestales y de seguros chilenas en el manejo de los riesgos de incendios forestales. El estudio analiza cómo las formas jerárquicas y mercantiles de gestión de riesgos se complementan entre sí y, paralelamente, limitan el uso de los conocimientos sobre el riesgo. Resulta evidente que cuando falta un conocimiento sofisticado, los actores adoptan estrategias para evitar el riesgo, en lugar de invertir colectivamente en los procesos de aprendizaje. La cuarta sección interpreta estas prácticas de gestión del riesgo como una descontextualización del riesgo y del conocimiento sobre los incendios forestales que se contrapone a un enfoque más colectivo de la gobernanza del riesgo. Finalmente, el articulo sugiere y promueve una perspectiva explícitamente regional de la gobernanza de los riesgos de incendios forestales en Chile.

\section{Los desafíos de la gestión de las incertidumbres económicas}

La noción de riesgo explica el contraste entre realidad y posibilidad (Hacking, 1990) y se refiere a un futuro que sólo es visible a través de la mirada del presente. Quienes asumen riesgos saben que sus decisiones van acompañadas de varias consecuencias posibles, pero sólo cuando el riesgo se materializa realmente en pérdidas son conscientes de sus verdaderas características y consecuencias. Mientras tanto, las ideas y percepciones del riesgo que circulan en la sociedad son continuamente conformadas e incluso manipuladas estratégicamente por el conocimiento discursivo del riesgo (Martin et al, 2009; González-Hidalgo \& Zografos, 2017). Los riesgos son fenómenos reales que se construyen socialmente y se amplifican discursivamente. Por lo tanto, es necesario comprenderlos, evaluarlos y gestionarlos por medio del uso de conocimiento sofisticado, lo que también exige un alto nivel de conocimientos sobre gobernanza (Rosa et al, 2014).

Desde el punto de vista económico, la imprevisibilidad del futuro no amerita preocupación. Los riesgos forman parte de los proyectos empresariales y prometen rendimientos superiores a la media. Quien logre controlar los riesgos mejor que otros prosperará en el mercado (Knight, 1982; Clark, 2018). Siguiendo esta línea argumental, es fácil ignorar que las decisiones individuales sobre los riesgos en la economía suelen ir acompañadas de efectos externos negativos para otros actores sociales. El riesgo de incendios forestales no es una excepción. Ciertas prácticas en el manejo de plantaciones forestales, por ejemplo, que se asocian con diferentes grados de probabilidad de accidentes, pueden desencadenar incendios forestales que, bajo ciertas condiciones climáticas, pueden propagarse rápidamente desde su lugar de origen a las áreas vecinas e incluso poner en peligro los asentamientos humanos (Bottaro et al, 2018). Muchos pueden sufrir pérdidas, incluso si en un principio no tomaron ninguna decisión sobre el riesgo. Mientras que las pérdidas económicas debidas al riesgo pueden ser fácilmente justificadas por los gestores de riesgo en retrospectiva -uno simplemente se refiere al conocimiento limitado sobre el riesgo que estaba disponible en el momento de la decisión y asegura el mejor manejo precautorio posible 
del mismo (Luhmann, 2006)- otros actores sociales tienen mayores dificultades para explicar sus posiciones. Las responsabilidades sociales en la asignación de riesgos a menudo no se aclaran.

El conocimiento incompleto de los riesgos, los efectos secundarios no deseados y otros "unknown unknowns"2 (Beck, 2006: 335) son desafíos fundamentales para la gobernanza de los riesgos. En última instancia, la forma en que se comunican los conocimientos sobre el riesgo determina el éxito o el fracaso de las prácticas de gestión y gobernanza del riesgo. Sin embargo, ¿cuáles son exactamente las diferencias entre ellas?

La gestión de riesgos se centra en la evaluación de los riesgos técnicos, con el objetivo de orientar y legitimar los procesos de toma de decisiones a nivel gerencial de una empresa y la coordinación de todos los recursos para hacer cumplir los objetivos de esta (Soin \& Collier, 2013; Lundqvist, 2015). Desde el punto de vista de una empresa, el riesgo se considera técnicamente como un factor de coste, en el que las probabilidades de que se ocurran incidencias dañosas se ponderan con el importe de la pérdida esperada (Knight, 1921). La gestión de riesgos calcula los riesgos utilizando datos históricos y cuantificables. Los sofisticados modelos de riesgo permiten decisiones individuales tomadas por expertos con el objetivo de reducir la vulnerabilidad de la empresa. La gestión del riesgo es de corto a medio plazo, dependiendo de la fiabilidad de los modelos de riesgo disponibles.

La perspectiva de la gobernanza del riesgo amplía el alcance de los actores involucrados en la gestión del riesgo y sus relaciones entre sí. Desde una perspectiva social, la gobernanza, en términos generales, coordina las acciones colectivas de actores legalmente independientes hacia el logro de objetivos comunes. Se extiende más allá del alcance de una autoridad única y requiere la negociación entre intereses particulares (Glückler et al, 2019). En concreto, la gobernanza del riesgo abarca los procesos que conducen a decisiones colectivamente vinculantes y al establecimiento de normas y prácticas de gestión del riesgo legitimadas (Renn, 2008; Van Asselt \& Renn, 2011). El interés científico en la gobernanza del riesgo apunta a analizar las estructuras institucionales, los procesos políticos y las constelaciones de poder que orientan y restringen las actividades de los distintos actores de la sociedad con el fin de regular, reducir o controlar los problemas colectivos de riesgo (Crouch \& Keune, 2012). Los desafíos de la gobernanza del riesgo están claramente asociados a la complejidad, ambigüedad y/o incertidumbre del conocimiento sobre el riesgo (Rosa et al, 2014). Solo en casos excepcionales se puede describir y calcular el riesgo de forma exhaustiva mediante correlaciones lineales. La complejidad, la ambigüedad y la incertidumbre son atributos de los riesgos que, si se distinguen empíricamente, implican mecanismos de gobernanza diferentes. La gobernanza del riesgo descubre y negocia interpretaciones disímiles del riesgo y crea nuevos conocimientos sobre el mismo para hacer frente a correlaciones inconclusas y desconocidas. Así, la gobernanza es un proceso de aprendizaje colectivo con un horizonte temporal a largo plazo, que se centra en soluciones colectivas específicas para cada contexto con el objetivo de aumentar la resiliencia de los actores sociales.

¿Cómo pueden complementarse positivamente las prácticas de gestión y gobernanza de riesgos en el contexto de los incendios forestales? ¿Quizás se interfieran mutuamente por su inconmensurable manejo del conocimiento sobre el riesgo? El siguiente artículo quiere llamar la 
atención sobre estas preguntas irresueltas y aplicarlas al caso empírico del sector forestal chileno que esta confrontado con el riesgo de incendios forestales.

\section{Metodología}

Para responder a las preguntas mencionadas, el presente artículo recopila los aportes de varios estudios empíricos sobre los riesgos en la industria forestal y maderera chilena, que se realizaron entre 2014 y 2018 como parte de diferentes cursos de seminario en el programa de magister en Gobernanza de Riesgos y Recursos ofrecido por el Heidelberg Center para América Latina. En los cursos se contrastaron los conocimientos teóricos sobre la gobernanza del riesgo con los esfuerzos prácticos de los agentes económicos para hacer frente a los fenómenos de riesgo real. Desde el principio, las investigaciones empíricas tuvieron como objetivo documentar las percepciones de riesgo de los diferentes actores expuestos a los incendios forestales y analizar sus esfuerzos colectivos para enfrentarlos a nivel regional. Curiosamente, los incendios forestales en Chile rara vez son percibidos como un riesgo colectivo por los actores encuestados. Predominan los esfuerzos individuales para minimizar las pérdidas económicas.

Dado que el riesgo de incendio forestal no puede evaluarse únicamente mediante hipótesis deterministas o probabilísticas, una triangulación de diferentes métodos de investigación cualitativa demostró ser apropiada y eficaz para la recopilación de los datos empíricos: i) En primer lugar, el documento se basa en una amplia evaluación de la literatura científica sobre el riesgo de incendios forestales en Chile. De esta manera, se pudo comprender, por un lado, la complejidad interdisciplinaria fundamental de las relaciones entre las causas y los efectos de los incendios forestales. Por otro lado, se podría comprender la forma en que se reduce y generaliza el conocimiento científico en los modelos contemporáneos de riesgo de incendios forestales. (ii) En segundo lugar, la revisión de la literatura se complementó con un análisis del contenido de los argumentos discursivos sobre las causas y efectos de los incendios forestales en Chile, tal como se discuten en revistas especializadas como Lignum, Revista Mundo Forestal, Revista CIFOR o la prensa en general ${ }^{3}$. En el análisis del contenido se incluyeron más de 250 artículos de prensa y reportajes sobre los incendios forestales ocurridos en Chile entre 2008 y 2018. Esto no solo proporcionó información adicional sobre las particularidades específicas regionales del riesgo, sino que también confirmó una amplia gama de explicaciones fuertemente generalizadas sobre el fenómeno de los incendios forestales, que indican el uso discursivo de los conocimientos sobre el riesgo (van Dijk, 2014). iii) En tercer lugar, se incluyó en el presente estudio una evaluación de los informes anuales de las grandes empresas forestales chilenas, así como de las estadísticas oficiales sobre incendios forestales (CONAF, 2018; INFOR, 2018) para destacar la variabilidad temporal y la diversidad regional del riesgo. Por un lado, las estadísticas muestran la magnitud cuantitativa de los incendios forestales catastróficos que son eventos muy específicos. Por otro lado, diferencian entre los actores afectados (propietarios de bosques naturales versus propietarios de plantaciones forestales de diferente tamaño). iv) Finalmente y sobre todo, el análisis se basa en

\footnotetext{
Entre ellos se incluyen periódicos nacionales como www.elmercurio.com; www.latercera.com; www.elmostrador.cl; www.cnnchile.com, pero también medios de comunicación regionales como www.diarioelcentro.cl; www.redmaule.com; www.diarioconcepcion.cl; www.biobiochile. cl. Sólo se analizaron los artículos en línea de estos periódicos. También se incluyeron en la investigación portales críticos de noticias en línea como www.terram.cl; http://www.laizquierdadiario.cl; www.mapuexpress.org; www.mapuexpress.org
} 
25 entrevistas guiadas de expertos con actores económicos, gestores de riesgos y personas en situación de riesgo en las regiones forestales chilenas (principalmente en las regiones del Maule y del Biobío). Las entrevistas se llevaron a cabo como entrevistas abiertas y basadas en guiones.

Precisamente porque el riesgo de incendio forestal es percibido de forma diferente por los diferentes actores en función de sus experiencias contextuales y es evaluado y comunicado de forma diferente en función del uso de los métodos de cálculo del riesgo, es necesario estudiar a los expertos en riesgos y sus interacciones entre sí. El conocimiento del riesgo diferenciado contextualmente influye en el manejo del riesgo de los actores (Müller-Mahn et al, 2018). En última instancia, también determina las posibilidades de percepción colectiva del riesgo y los consiguientes esfuerzos en los procesos de gobernanza.

\section{¿Complejidad, ambigüedad, incertidumbre? Riesgos de incendios forestales en Chile}

Los incendios forestales naturales son típicos de las zonas de clima mediterráneo, caracterizadas por primaveras suaves y lluviosas, seguidas de veranos secos. También son un fenómeno natural en varias regiones del centro-sur de Chile (McWethy et al, 2018). Los incendios forestales son eminentemente espaciales: tienen un lugar de origen claramente definido desde el que se extienden por leyes geofísicas a espacios cercanos. Hoy en día, los incendios forestales son principalmente fenómenos provocados por el hombre. En muchos casos, son causados por descuidos, accidentes o incluso incendios arbitrarios (O'Flanagan, 1997). En este sentido, representan externalidades negativas de la expansión de las actividades humanas.

La figura 1 resume la frecuencia de eventos de incendios forestales en Chile desde la década de 1990. En promedio, se producen casi 6.000 incendios forestales por año y se pierden unas 10 hectáreas de terreno por cada incendio. Dos tercios de las zonas afectadas son paisajes naturales, como bosques naturales, matorrales y pastizales. Sin embargo, también los bosques de plantación se ven afectados en Chile, y esta tendencia va en aumento (Julio, 2014). Más recientemente, una fase de sequía que duró varios años ocasionó los incendios forestales más grandes de la historia de Chile (González et al, 2018). En la figura 1 se destaca la dimensión destructiva de la "tormenta de fuego" (Gobierno de Chile, 2017) en términos de áreas quemadas.

Hasta cierto punto, los riesgos de incendios forestales pueden evaluarse con correlaciones lineales que se refieren a las condiciones físico-geográficas (Castillo et al, 2013). En la terminología de las autoridades forestales, que controlan el riesgo de incendios, se habla, por ejemplo, de la "regla del 30-30-30". La norma dice que en el caso de temperaturas superiores a 30 grados, las corrientes de viento superiores a 30 kilómetros por hora y una humedad relativa del aire inferior al $30 \%$ aumenta significativamente el riesgo de incendios. Es indiscutible que bajo condiciones climáticas extremas de temperatura, viento y humedad, un incendio forestal puede expandirse muy rápidamente. Sobre la base de estos datos, las autoridades pueden publicar alertas de riesgo a tiempo para concienciar a la población sobre el peligro potencial. La topografía tiene otra influencia directa en la velocidad de propagación de los incendios forestales. Cuanto más pronunciada es la pendiente, mayor es la inclinación de las llamas, lo que aumenta el desarrollo de calor 
en el suelo y permite que la biomasa se queme más rápida e intensamente. Este conocimiento es importante para el combate del fuego.

Figura 1: Evolución de los incendios forestales en Chile y daños relacionados

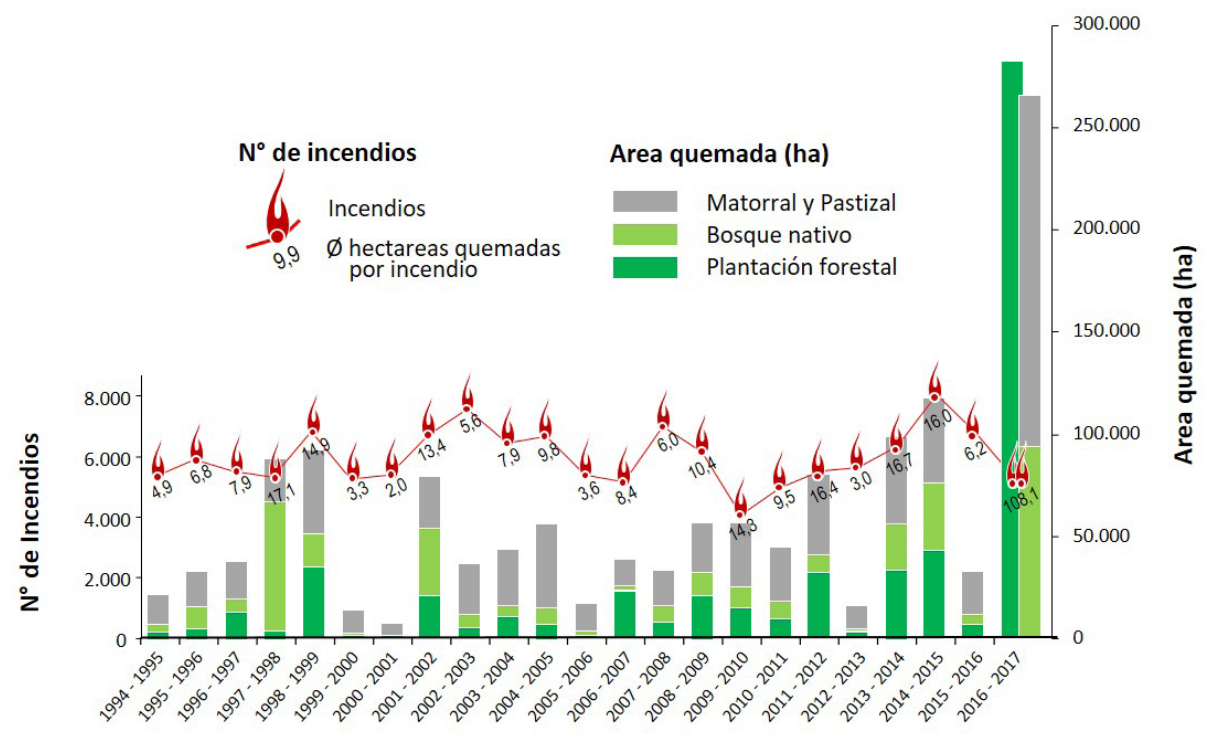

Fuente: Elaboración propia con datos de la CONAF (2018)

Sin embargo, los factores naturales por sí solos no pueden explicar plenamente la magnitud de los incendios forestales en Chile (Gómez-González et al, 2019). Pueden explicar su propagación y, por lo tanto, la dimensión dañina del riesgo. Aun así, las causas de los incendios suelen tener un trasfondo antropogénico. Los análisis de riesgo, por ejemplo, demuestran una alta correlación entre los eventos de incendios forestales en las proximidades de los asentamientos humanos y a lo largo de las redes de carreteras (Martínez-Fernández et al, 2004). Allí, el hombre es imprudente en su tratamiento de la naturaleza. Las formas de uso de la tierra y, en particular, el tipo de composición de las especies arbóreas en los bosques económicamente explotados de Chile también influyen en la dimensión del riesgo. Esta relación, sin embargo, es menos concluyente (ver por ejemplo el polémico debate publicado en Mundo Forestal, 2017). Para entender el fenómeno de los incendios forestales en Chile, se deben considerar conjuntamente los factores físicos y antropogénicos contextuales. La investigación interdisciplinaria es indispensable, lo que, sin embargo, conduce a desafíos epistemológicos de complejidad, ambigüedad e incertidumbre.

Es muy común en la literatura describir riesgos con estas tres características (Knight, 1921; Perrow, 1986; Ericson \& Doyle, 2004; Cabantous, 2007; Brugnach \& Ingram, 2012; Johansen \& Rausand, 2015; Ilin \& Varga, 2015; Müller-Mahn et al, 2018), que pueden distinguirse en pocas palabras de la siguiente manera (véase también Rosa et al, 2014): La complejidad caracteriza una condición 
en la que es difícil identificar y cuantificar exactamente las posibles relaciones causales y los efectos adversos específicos de la toma de decisiones sobre el riesgo. La ambigüedad corresponde al fenómeno de que los distintos agentes conocen el riesgo de manera diferente, lo que da lugar a una variabilidad de las interpretaciones legítimas del riesgo con respecto a las observaciones y evaluaciones de datos idénticos. La incertidumbre se refiere al conocimiento desconocido sobre ciertas relaciones causales. Eso incluye no saber lo que no sabemos. El conocimiento científico disponible y las experiencias que circulan en Chile sobre el riesgo de incendios forestales pueden distinguirse por estas tres características.

\section{Complejidad: correlaciones multidireccionales entre factores físicos y antropogénicos}

En primer lugar, es el sector forestal, con su forma específica de organizar las actividades económicas en el espacio (Clapp, 1995; Gatica, 2012), el que crea vínculos complejos entre los factores antropogénicos y naturales del riesgo de incendios. Las empresas forestales chilenas, por ejemplo, deciden sobre la composición de las especies de árboles en sus plantaciones, donde favorecen especies exóticas como el eucalipto o el pino, ya que se adaptan a las condiciones climáticas secas, crecen rápidamente y, por lo tanto, son muy rentables económicamente. Actualmente en Chile, los pinos representan el $58 \%$ de toda el área de plantación, mientras que los eucaliptos representan el $36 \%$ (INFOR, 2018). Sin embargo, como ambas especies consumen más agua que los árboles nativos, pueden intensificar las sequías a escala microgeográfica y aumentar el riesgo de incendios (Little et al, 2009). No obstante, la gestión industrial de las plantaciones puede reducir significativamente la dimensión del riesgo. En su búsqueda de mayores rendimientos, las forestales eliminan la "biomasa innecesaria" de las plantaciones y controlan así importantes aceleradores de incendios. Al invertir en torres de vigilancia que colocan estratégicamente dentro de sus territorios, organizan un sistema de monitoreo interconectado que permite detectar a tiempo los incendios forestales (Tapia \& Castillo, 2014). Las propias brigadas de bomberos de las empresas pueden llegar rápidamente a la fuente de un incendio a través de senderos forestales. Entonces, si la probabilidad de incendios permanece constante, el riesgo en las plantaciones forestales se reduce. Aun así, la complejidad surge porque muchos de los factores que aumentan y reducen el riesgo no se pueden medir juntos de manera precisa. Este es, por cierto, un problema metodológico para muchos modelos de predicción de riesgos. Para que estos modelos funcionen, deben elegirse supuestos normativos sobre la ponderación de los factores individuales.

El cuadro $N^{\circ} 1$ resume las actividades forestales y el riesgo de incendios regionales en Chile e ilustra la complejidad de las relaciones entre los factores físicos y antropogénicos. En Chile, el riesgo climático de incendios forestales debería disminuir en las regiones forestales meridionales, ya que estas tienen temperaturas medias más bajas a lo largo del año y, en general, registran mayores precipitaciones (Altamirano et al, 2013). Un estudio del cuadro $N^{\circ} 1$, sin embargo, revela que los incendios forestales en el sur son más frecuentes en términos absolutos y medidos en términos de superficie forestal disponible que en las regiones forestales del norte. También es un hecho que en las regiones del Biobío y la Araucanía, los incendios forestales son mucho más frecuentes en las plantaciones forestales que en los bosques naturales. Sin embargo, los incendios en las plantaciones son significativamente menos destructivos que los incendios que afectan a los bosques nativos. 
Cuadro $N^{\circ}$ 1: Actividades forestales e incendios forestales en Chile

\begin{tabular}{|c|c|c|c|c|}
\hline & $\begin{array}{l}\text { O'Higgins } \\
\text { (VI) }\end{array}$ & $\begin{array}{l}\text { Maule } \\
\text { (VII) }\end{array}$ & $\begin{array}{c}\text { Biobío } \\
\text { (VIII) }\end{array}$ & $\begin{array}{l}\text { Araucania } \\
\text { (IX) }\end{array}$ \\
\hline Áreas forestales 2014 [ha] & 586.615 & 833.227 & 1.695 .082 & 1.447 .635 \\
\hline Plantaciones forestales 2014 [ha] & 127.306 & 448.513 & 926.530 & 483.482 \\
\hline $\begin{array}{l}\text { Eventos anuales de incendios } \\
\text { ( } \varnothing \text { anual 2003-2016) [número absoluto] }\end{array}$ & 222 & 437 & 2.499 & 1.006 \\
\hline Incendios forestales por 1.000 hectáreas & 0,38 & 0,52 & 1,47 & 0,69 \\
\hline [\%] de los eventos que afectan a plantaciones & 1,4 & 15,5 & 59,1 & 56,0 \\
\hline $\begin{array}{l}\text { Daño anual (total bosque) } \\
\text { ( } \varnothing \text { anual 2003-2016) [ha] }\end{array}$ & 7.927 & 7.001 & 13.487 & 5.996 \\
\hline Daños en bosques nativos [ha por incendio] & 35,1 & 17,7 & 11,1 & 9,3 \\
\hline Daños en plantaciones [ha por incendio] & 38,6 & 17,4 & 1,9 & 3,3 \\
\hline
\end{tabular}

Fuente: Elaboración propia con datos estadísticos de CONAF (2018)

La búsqueda de explicaciones complejas sobre la magnitud de los daños en Chile lleva a la pregunta de quién invierte en la extinción de incendios en primer lugar y quién decide qué fuentes de fuego se extinguen primero cuando se producen varios incendios en paralelo. Dado que la lucha contra los incendios forestales en Chile está sujeta en gran medida a un mercado en el que las empresas privadas de extinción de incendios ofrecen sus servicios -y en el que el valor económico de los recursos madereros determina a menudo la disponibilidad de las brigadas de bomberos-, es comprensible que los incendios forestales provoquen interpretaciones críticas del modelo neoliberal de silvicultura (Barton \& Román, 2012; Reyes \& Nelson, 2014). En relación con estas discusiones, entran en juego las características ambiguas del riesgo de incendios forestales en Chile.

\section{Ambigüedad: la coexistencia de varias explicaciones igualmente lógicas}

Es a través del conocimiento discursivo (van Dijk, 2014) que los riesgos se construyen socialmente. El conocimiento geográfico de las complejas relaciones de riesgo da sustancia explicativa a estos discursos. "Los riesgos solo adquieren influencia en el mundo social en la medida en que se comunican" (Renn, 2008: 57).

En Chile circulan múltiples explicaciones discursivas sobre los orígenes y la peligrosa eficacia de los incendios forestales. Ciertamente se alimentan de la ambigüedad del conocimiento del riesgo. Por un lado, los discursos reflejan las amenazas percibidas por diferentes actores sociales (Mermoz et al, 2005). Por otra parte, la ambigüedad resultante se debe a las dificultades lingüísticas para articular el riesgo. Aquellos que han experimentado el riesgo de primera mano, es decir, aquellos que han sufrido pérdidas, a menudo describen sus experiencias de manera que se maximice la influencia sobre los responsables de la toma de decisiones políticas para que sirvan a su propio beneficio (Farré, 2005). Esto puede llevar a interpretaciones relacionadas con los incendios forestales que son artificialmente exageradas e intencionalmente falsas. Es por ello 
que la ambigüedad puede entenderse como una función del conflicto sobre las preferencias, la intersubjetividad del discurso y la incertidumbre sobre los aspectos técnicos de la comunicación de riesgos (Hanson \& Kysar, 1999; Johansen \& Rausand, 2015). La consiguiente ambigüedad de los discursos no se refiere tanto a un futuro incierto como a la incertidumbre relacionada con las experiencias pasadas y presentes.

En todas las regiones chilenas, las principales causas de los incendios forestales son los accidentes y el descuido (58\%). Sin embargo, casi uno de cada tres incendios es causado por la delincuencia, es decir, por encendidos causados intencionadamente, mientras que el $15 \%$ de los incendios permanecen en la oscuridad. Son sobre todo las causas desconocidas e intencionadas de los incendios forestales las que se reportan y especulan en detalle en la prensa chilena (Millones, 2017; ver también la reseña de Aylwin, 2017).

Cuadro $N^{\circ} 2$ : Causas de los incendios forestales en Chile ( $\varnothing$ anual 2003-2016)

\begin{tabular}{|l|r|r|r|r|}
\hline & $\begin{array}{c}\text { O'Higgins } \\
\text { (VI) }\end{array}$ & \multicolumn{1}{c|}{$\begin{array}{c}\text { Maule } \\
\text { (VII) }\end{array}$} & \multicolumn{1}{c|}{$\begin{array}{c}\text { Biobío } \\
\text { (VIII) }\end{array}$} & $\begin{array}{c}\text { Araucania } \\
\text { (IX) }\end{array}$ \\
\hline $\begin{array}{l}\text { Eventos anuales de incendios } \\
\text { (Ø anual 2003-2016) [número absoluto] }\end{array}$ & 222 & 437 & 2.499 & 1.006 \\
\hline Causas: Accidentes [\%] & 67,3 & 68,9 & 27,5 & 37,0 \\
\hline Causas: Actividades agrícolas y forestales [\%] & 14,3 & 15,5 & 8,4 & 12,0 \\
\hline Causas: Incendios intencionales [\%] & 14,4 & 11,0 & 35,7 & 46,5 \\
\hline Causas desconocidas [\%] & 3,8 & 4,2 & 28,2 & 3,9 \\
\hline
\end{tabular}

Fuente: Elaboración propia con datos estadísticos de CONAF (2018)

Según las estadísticas oficiales, se observa una distribución regional desigual de las causas de los incendios, especialmente en los incendios provocados intencionadamente. Esto significa que dependiendo de a quién se le pregunte y de los antecedentes históricos y regionales, se establecen repetidamente las siguientes interrelaciones causales:

(i) Algunos sostienen que los incendios forestales en Chile son causados por los propios propietarios de los bosques con el fin de eludir las normas de planificación territorial existentes (Caviedes, 2017). En las proximidades de las zonas urbanas, por ejemplo, después de un incendio, se especula que las zonas forestales quemadas pueden ser designadas como terrenos urbanos en desarrollo. Esta es una realidad en muchas partes del mundo (Salvati \& Ranalli, 2015). En Chile, estas causas sólo se discuten narrativamente en público, ya que las acusaciones a la rápida pueden tener consecuencias legales.

(ii) Los incendios forestales intencionales también se interpretan como una estrategia deliberada de expansión forestal. Tales interpretaciones, en particular, surgen en comunidades donde los propietarios de tierras reforestan sus bosques naturales quemados como plantaciones foresta- 
les (Gerber, 2011). En Chile, desde la entrada en vigor en 2008 de la nueva "ley de recuperación de los bosques nativos" (CONAF, 2008), se prohíbe a los propietarios forestales convertir los bosques naturales en plantaciones forestales con especies exóticas. Sin embargo, después de un incendio se permite la reforestación, por ejemplo, en forma de plantaciones con especies endémicas. De esta manera, los recursos maderables naturales se transforman y se hacen accesibles para el uso industrial (Moreno del Valle, 2015).

iii) Se argumenta además que los propios propietarios forestales queman sus bosques para beneficiarse de los seguros forestales que han adquirido anteriormente (Focacci, 2017). Este argumento en su forma simple parece plausible, pero suele ser engañoso y requiere explicaciones detalladas y contextualizadas: Los aseguradores son generalmente muy conscientes del problema del llamado riesgo moral (Stiglitz, 1983), es decir, la posible quema deliberada de un bosque que está asegurado. Por lo tanto, las compañías de seguros diseñan los contratos de tal manera que el asegurado siempre asume un riesgo parcial (Agroseguros, 2018). Los deducibles, por ejemplo, reducen los incentivos para cometer fraude. Sin embargo, estos incentivos pueden ser muy diferentes para los propietarios de bosques que están al borde de la ruina, por ejemplo, en los casos en que una calamidad de insectos redujo significativamente el valor de un bosque en pie (véase, por ejemplo, la observación de los hechos específicos en Mapucheexpress, 2017). Mientras las condiciones contractuales que las empresas forestales negocien con el sector de seguros no sean transparentes públicamente, continuará la especulación discursiva y ambigua sobre las motivaciones de los pirómanos.

iv) En algunos casos, los incendios intencionales en Chile son producto del descontento social. En las regiones del Maule, Biobío y Araucanía, por ejemplo, parece que están directamente dirigidos contra las grandes empresas forestales que con sus plantaciones marcan el uso de la tierra y dictan las principales actividades económicas de la región (Van Holt et al, 2016). Una opinión pública muy difundida es que, en la región de la Araucanía en particular, miembros del pueblo mapuche habrían prendido fuego a modo de protesta contra los déficits políticos y/o socioeconómicos. Los incendios forestales parecen haberse convertido en el símbolo de su conflicto con el Estado chileno (Montalba-Navarro \& Carrasco, 2003; Rojas \& Miranda, 2015). El sur de Chile experimentó tardíamente, después de la colonización española, con la nacionalización del país, una apropiación y valorización capitalista de la tierra que crea gente vulnerable y que aún continúa hoy en día (Latorre \& Rojas, 2016). Pero incluso en este caso las circunstancias de los incendios forestales no están del todo claras, ya que algunos de los incendios en la región de la Araucanía fueron causados por el sector privado e incluso por funcionarios públicos buscando culpar a la comunidad mapuche por el delito y así mantener vivo el conflicto de manera discursiva (Sepúlveda, 2013; Seguel, 2018). De esta manera, los incendios sirven para justificar retrospectivamente la expansión forestal (González-Hidalgo \& Zografos, 2017). La coexistencia de estos diferentes discursos enfrenta a los actores con problemas en la toma de decisiones. Oscurecen los verdaderos motivos de los actores tras los incendios, lo que lleva a características inciertas de riesgo.

\section{Incertidumbre: Amplificación social oculta y autofortalecimiento del riesgo}

Los riesgos modernos ya no originan sus causas o efectos en un solo lugar. Además, pueden tener un largo período de latencia. Las personas vulnerables al riesgo y los responsables del mis- 
mo no son localizables ex-ante (Beck, 1998). Para los actores políticos, esta es la principal fuente de incertidumbre: "son precisamente las incógnitas desconocidas las que provocan conflictos de largo alcance sobre la definición y construcción de reglas y responsabilidades políticas con el objetivo de prevenir lo peor" (Beck, 2006: 335). A continuación, se centrará la atención en las características sistémicas de los riesgos inciertos de incendios forestales. Dependiendo del contexto local y regional, la ocurrencia de incendios forestales en Chile añade un autofortalecimiento al riesgo:

(i) Existen, por ejemplo, casos de incendios forestales en Chile, especialmente en las interfaces de los asentamientos, que son causados deliberadamente por jóvenes. Investigaciones de la fiscalía revelaron que ellos iniciaron el incendio "[...] para experimentar una vez más el espectacular uso de aviones o helicópteros para extinguir incendios" (entrevista con bomberos forestales en Constitución/Maule, 2015). La legítima preocupación por este tipo de origen de los incendios se refleja en la normativa que regula los cuerpos de bomberos. Las normas estipulan que las misiones de helicópteros en caso de incendio deben restringirse a áreas fuera de los asentamientos (Vélez, 2009). Se puede interpretar que esto es para evitar que las experiencias de incendios pasados causen nuevos incendios en el futuro.

(ii) Otro aspecto sistémico del riesgo se evidencia en la apropiación estratégica del efecto simbólico del fuego por parte de grupos muy diferentes (Segovia et al, 2018). Como imaginario social, los incendios forestales adquieren incluso importancia y eficacia para los miembros de la sociedad que viven lejos de las zonas forestales. Los incendios transmiten imaginarios socio-críticos. Para algunos, representan la lucha de los vulnerables contra la alianza neoliberal del Estado con la agricultura industrial y la silvicultura. Para otros, adquieren importancia al justificar que el Estado se pone del lado e impone un control más estricto sobre los recursos periféricos. Diferentes grupos esperan ventajas individuales para su negocio de los incendios forestales. El riesgo trasciende los límites de los sistemas cuando sus efectos se extienden desde el paisaje forestal hasta las esferas económicas y políticas (Beck, 2006).

Los riesgos sistémicos son inciertos hasta que se materializan y, por lo tanto, no pueden calcularse ex-ante (Ilin \& Varga, 2015). Para hacerles frente, es necesario reajustar la percepción del riesgo. En el primer caso, esto se hizo, como se ha descrito, mediante nuevas normas que limitan el uso de helicópteros. En el segundo caso, sin embargo, el efecto sistémico del riesgo sigue estando fuera del alcance de los gestores de riesgos. Independiente de sus reacciones contextuales, estos no pueden impedir que otros actores traten continuamente de explotar el efecto simbólico de los incendios forestales.

Como se deduce por lo anterior, los desafíos epistemológicos de complejidad, ambigüedad e incertidumbre del riesgo de incendio forestal surgen del hecho de que las tres características están estrechamente entrelazadas y conducen a ensombrecer las relaciones. En este contexto, es comprensible que la gestión del riesgo por lo general se dirija a simplificar y separar los atributos individuales. Esto permite a los gestores de riesgos transformar la ambigüedad o la incertidumbre en complejidad, que luego puede estructurarse y manejarse mediante el uso de modelos técnicos de riesgo. 


\section{La complementariedad de las prácticas de gestión de riesgos}

Los conocimientos teóricos de la Nueva Economía Institucional (Richter \& Furubotn, 1997), que se aplican ampliamente en las prácticas de gestión del riesgo financiero (Ericson \& Doyle, 2004), proclaman que los mercados y las jerarquías son, en cierto sentido, formas de gobernanza idealmente opuestas para la coordinación de la interacción económica. Las características de una transacción, así como el grado de distribución asimétrica de la información entre los agentes económicos, determinan si los pedidos jerárquicos a través de las rutinas de la empresa o los procesos de fijación de precios basados en el mercado en condiciones competitivas proporcionan soluciones de coordinación de coste eficiente para la transacción. Curiosamente, como lo demuestran las prácticas de gestión de riesgos en Chile, las formas de gestión jerárquicas y basadas en el mercado no son necesariamente opuestas entre sí, sino que se apoyan mutuamente. Una gestión de riesgos organizada jerárquicamente parece ser incluso el requisito previo para el acceso a las soluciones del mercado de seguros.

Para entender bien la lógica complementaria detrás de estas prácticas, es importante señalar que las empresas forestales y las aseguradoras solo difieren ligeramente en su interpretación basica del riesgo y la incertidumbre. Ambos actores son capaces y están dispuestos a manejar el riesgo que pueden calcular, y ambos rechazan la incertidumbre en la gestión del riesgo. Entonces, ¿cómo puede configurarse un mercado de seguros contra incendios forestales si los riesgos preferidos e indeseables de ambos actores son muy parecidos? Por supuesto, depende de las pequeñas diferencias en el manejo de los riesgos, y hay que mirar las estrategias de evitación de la incertidumbre de ambos actores para entender el carácter complementario del manejo del riesgo de incendios forestales en Chile.

Para las compañías de seguros, los riesgos calculados representan sin duda una actividad principal. Los aseguradores pueden diversificarlos con la ayuda de la ley de los grandes números, que establece que la realidad empírica (temporal y espacial) del riesgo puede agregarse en valores medios (Hacking, 1990). Las empresas forestales también tienen una opinión positiva sobre los riesgos. Si se gestionan correctamente, los riesgos prometen rendimientos superiores a la media y ventajas competitivas (Lundqvist, 2015). La incertidumbre, es decir, el riesgo incalculable, es algo que ninguno de los dos actores está buscando activamente. Tanto las compañías forestales como las aseguradoras están tratando de externalizar o evitar completamente los riesgos que son raros (y al mismo tiempo muy destructivos), altamente específicos e inciertos. Las empresas, en particular, tienen temor de los riesgos que son asumidos por otros y que "ponen en peligro" el éxito de sus propios negocios en el sentido de una externalidad negativa (Luhmann, 2006). Las compañías de seguros, a su vez, hacen todo lo posible para evitar el riesgo moral de sus clientes, lo que puede llevar incluso a la situación de que no exista una oferta de mercado para los seguros en absoluto (Hellwig, 1983; Stiglitz, 1983). Esta línea de argumentación encaja con el enfoque del costo de transacción, que estipula solo al control jerárquico para tratar los riesgos inciertos (véase también Knight, 1921). ¿Cómo surge el mercado de seguros cuando los participantes del mercado comparten el deseo común de renunciar o evitar la incertidumbre? La respuesta tiene que ver con las diversas prácticas de estandarización y categorización de riesgos para reducir su complejidad, ambigüedad e incertidumbre. 
La gestión de riesgos en general tiene por objeto enumerar objetivamente todos los resultados probables a fin de reducir posteriormente la incertidumbre a una lista de eventos probables (Beckert, 2016). Por un lado, se incluyen las prácticas de disociación y simplificación del riesgo a través de la homogeneización de condiciones contextuales seleccionadas. Estas prácticas se utilizan principalmente en empresas forestales donde la gerencia coordina rutinas de gestión de riesgos descentralizadas y centralizadas (Perrow, 1986; Becker \& Knudsen, 2005). Las compañías de seguros, por su parte, aplican prácticas de estandarización y economización de riesgos, todas basadas en procedimientos de cuantificación matemática (Jarzabkowski et al, 2015).

\section{Gestión de riesgos en la silvicultura chilena}

En el sector forestal industrializado de Chile, las plantaciones forestales se manejan de una manera que minimiza tanto la probabilidad de un incendio forestal como la posibilidad de una expansión incontrolada. La homogeneización de las condiciones contextuales, la simplificación de las relaciones de riesgo y la desacoplación de ciertos elementos de riesgo van de la mano y se influyen recíprocamente: i) La simplificación del riesgo comienza por centrarse en determinadas causas de los incendios forestales. En particular, la causa principal de los accidentes puede ser minimizada a través de la capacitación de los empleados y de instrucciones claras y rutinarias. La orientación de la gestión de las plantaciones hacia economías de escala industriales es fundamental para la implementación de las rutinas, solo se necesitan unos pocos especialistas para controlar el cumplimiento de las mismas. ii) El desacoplamiento de las causas y los efectos de los incendios forestales se logra mediante inversiones específicas en infraestructura, como, por ejemplo, la formación de cortafuegos que frenan o detienen los incendios que han ocurrido. A menudo, los cortafuegos se crean estratégicamente en el curso de los cortes regulares. En este caso, las prácticas de manejo de plantaciones y las inversiones en infraestructura coinciden. Además, los caminos forestales son una inversión estratégica que sirve para la gestión de riesgos, ya que permiten el acceso rápido de los cuerpos de bomberos. iii) Por último, la homogeneización del espacio físico complementa el desacoplamiento y la simplificación de las relaciones de riesgo. Las grandes empresas forestales chilenas buscan una propiedad forestal consolidada y coherente. En los últimos 40 años, lo han logrado en gran medida a través de continuas adquisiciones de propiedad forestal. Solo unas pocas propiedades forestales privadas separan aún las plantaciones forestales de las grandes empresas. Las áreas forestales conectadas permiten economías de escala en la logística forestal. También pueden supervisarse mejor con torres de vigilancia de protección contra incendios, lo que a su vez contribuye aún más a el desacoplamiento de los riesgos, ya que los incendios pueden detectarse rápidamente una vez que han ocurrido.

Todas estas prácticas de gestión de plantaciones se basan en la combinación de lógicas de gestión de riesgos centralizadas y descentralizadas. También son evidentes múltiples referencias espaciales: la reducción de la complejidad a través de rutinas, por ejemplo, se planifica y coordina jerárquicamente de forma descendente desde la sede central de las empresas. Sin embargo, la implementación y el monitoreo de las rutinas en un lugar específico se lleva a cabo de manera descentralizada. Las torres de vigilancia son un nodo importante en la red de comunicación de riesgos. En caso de un incendio se comunican tanto a las unidades vecinas como a la sede de la empresa para que las intervenciones puedan ser elaboradas de manera estratégica y a tiempo. En caso de un desastre, las cadenas de mando de tipo militar se activan y toman el control. Los bomberos están coordinados centralmente pero pueden decidir localmente para poder reaccio- 
nar rápidamente a las condiciones cambiantes (Arnaldos et al, 2004). Con esta combinación de rutinas de gestión de riesgos centralizadas y descentralizadas, las empresas forestales están en una buena posición para reaccionar a las señales ambiguas de las regiones forestales.

\section{Gestión de riesgos en las compañías de seguros}

Para las compañías de seguros, la gestión de riesgos es principalmente una práctica de estandarización y categorización del riesgo a través de la cuantificación (Williamson, 1993). Dado que la cuantificación desconecta los datos existentes de las narrativas locales o de las historias generales que estabilizan el significado del riesgo en primer lugar, las prácticas de seguros deben crear sus propios significados de riesgo. Los precios en los mercados de seguros, por ejemplo, son un estándar para la cuantificación del riesgo. Comunican la magnitud del riesgo a terceros, como los clientes (Hayek, 1945; Kessler, 2015). Los mapas de riesgo proporcionan un marco de comunicación cuantificado similar (Dransch et al, 2010). A través de los mapas, las compañías de seguros señalan a sus clientes por qué las pólizas de seguros en las llamadas áreas de alto riesgo contienen una prima alta. Los mapas de riesgo dan sentido a los precios. Sin embargo, este tipo de comunicación de riesgos requiere en primer lugar un proceso de valoración (Aspers, 2009). La valoración del riesgo en las prácticas de gestión de riesgos de las compañías de seguros, como se ha mencionado anteriormente, se basa principalmente en modelos matemáticos y lógicas de correlación. Estos modelos son capaces de captar la complejidad de los aspectos y condiciones individuales que causan o amplifican los incendios forestales y descomponerlos en sus componentes individuales por medio de múltiples (e incluso espaciales) regresiones (Castro \& Chuvieco, 1998). Los modelos de riesgo espacialmente diferenciados permiten a las aseguradoras simular sus valores en riesgo.

Cuanto mejor logre la compañía de seguros descomponer la complejidad de los eventos de riesgo de incendio forestal en cadenas modeladas de causas y efectos lineales, mejor podrá diseñar y ofrecer diferentes tipos de contratos de seguro. Por un lado, las compañías de seguros siempre tienen la libertad de decidir si y dónde ofrecer una póliza de seguro a un cliente. Esto significa que las zonas en las que se producen incendios forestales con mucha frecuencia y por razones desconocidas pueden quedar excluidas del acceso al seguro. El mercado simplemente se raciona geográficamente (Hellwig 1983). Por otra parte, las compañías de seguros tienen a su disposición una fuerte herramienta de diseño de contratos: la llamada autoselección (Richter \& Furubotn, 1997). Al ofrecer a los clientes diseños de contrato alternativos que difieren, por ejemplo, en el importe del deducible en caso de siniestro, los aseguradores obtienen información detallada sobre la exposición al riesgo de un cliente. Al aceptar ciertos contratos y rechazar otros, el asegurado revela su actitud de riesgo y autoevalúa su exposición al riesgo. La auto-selección es parte de un proceso de negociación bilateral. En las negociaciones, los propios clientes se ofrecen a cumplir ciertas condiciones para bajar los precios de los seguros. Por ejemplo, cuanto mejor controle el riesgo la propia empresa forestal mediante la simplificación, la homogeneización y la disociación de sus efectos, más barata será la prima de seguro ofrecida.

\section{Prácticas de gestión de riesgos mutuamente complementarias y estrategias de evitación de riesgos}

Las prácticas de gestión de riesgos jerárquicas y orientadas al mercado se complementan entre sí al menos de dos maneras. Por un lado, esto puede verse en el hecho de que las prácticas 
de gestión forestal estimulan la innovación en los servicios de las aseguradoras. Sin economías de escala en el manejo de plantaciones industrializadas, el mercado de seguros contra incendios forestales en Chile habría sido demasiado pequeño, demasiado costoso en transacciones y posiblemente inexistente. Esto queda ilustrado, por ejemplo, por el hecho de que actualmente no existen pólizas de seguro disponibles para los bosques naturales utilizados económicamente. Faltan formas estandarizadas de calcular el valor de estos tipos de bosques. Por otro lado, la complementariedad surge más obviamente de la forma en que los aseguradores influyen en las normas de gestión forestal de las empresas forestales a través de la autoselección y el diseño de contratos. Los aseguradores dejan claro lo importante que es para ellos que los trabajadores forestales raleen regularmente las plantaciones (= simplificación de las correlaciones de riesgo), que las plantaciones aseguradas tengan un tamaño mínimo (= homogeneización del riesgo) y que se proporcionen torres de vigilancia y cortafuegos en el área de riesgo (= desacoplamiento de las correlaciones de riesgo). Estos requisitos se especifican directamente en los contratos de seguro, o bien se dan incentivos indirectos a las empresas forestales para que los cumplan mediante descuentos en las primas prometidas.

Los efectos autofortalecedores de las formas jerárquicas y de mercado de la gestión de riesgos también se derivan de las estrategias de evitación de la incertidumbre de los actores. Las empresas forestales, por ejemplo, quieren deshacerse del escenario poco probable, pero en principio posible, de una pérdida total de sus activos en caso incendios forestales catastróficos. El escenario de pérdida total no es aceptable para ellos, pero tampoco es calculable y por lo tanto incorpora las características de incertidumbre. Las grandes empresas forestales evitan esta incertidumbre transfiriéndola al sector de los seguros. Compran pólizas de seguro que incluyen deducibles altos. Como resultado, muchas pérdidas pequeñas y medianas serán cubiertas por las propias empresas forestales a pesar de haber invertido en seguros. "Incluso en el año de desastre 2017, los pagos de compensación de nuestras aseguradoras estaban por debajo de los gastos de las primas de seguro anuales. Todos nuestros contratos incluían deducibles elevados" (Entrevista 2018). Según la empresa forestal, es el deseo explícito de los accionistas de la empresa asegurar solo la incertidumbre de la pérdida total. Las pérdidas económicas menores debidas a incendios forestales, en cambio, se anticipan y son cubiertas por la propia empresa.

Lo que a las empresas forestales les parece un riesgo incierto incalculable, causado por terceros y, por lo tanto, entendido como un riesgo externo, es, a los ojos de las aseguradoras, complejo y cuantificable. Las compañías de seguros son capaces de absorber la incertidumbre de una pérdida total de un cliente porque pueden convertirla en un riesgo calculable y estadísticamente bajo de una pérdida total en todos sus clientes. Sin embargo, las aseguradoras conservan gran parte de su control sobre el riesgo explícitamente a través de sus propias estrategias para evitar la incertidumbre: evitan la incertidumbre mediante el racionamiento del mercado. Esta estrategia está orientada geográficamente de dos maneras. Por un lado, como ya se ha mencionado, excluyen los seguros en municipios con alto potencial de tensión política, es decir, en territorios donde el incendiarismo es una causa frecuente de incendios o donde las causas son simplemente desconocidas. Por otro lado, también excluyen áreas forestales específicas a pequeña escala que declaran no asegurables. Al evitar los llamados "riesgos de conglomerado" y los riesgos de contagio geográfico, un asegurador niega el acceso al mercado a los propietarios forestales situados en la proximidad geográfica inmediata de los bienes forestales ya asegurados. En una situación de entrevista en 2016, el ejecutivo de ventas de una compañía de seguros chilena se refirió a un mapa en la pared de su oficina, en el que los sitios de las áreas forestales actualmente asegura- 
das bajo su responsabilidad estaban marcados con banderas. Estas marcas daban al ejecutivo de ventas una indicación del área circundante donde no podía (o no debía) ofrecer ningún otro seguro contra incendios forestales. "Evito vender aquí porque un solo incendio forestal podría afectar a varios de mis clientes al mismo tiempo", explicó. En un mercado como el chileno, que se caracteriza por un número limitado de aseguradoras, esto lleva a la exclusión de los propietarios forestales dispuestos a comprar seguros. El racionamiento de los productos financieros es típico de los mercados de seguros oligopolísticos (Hellwig, 1983). Sin embargo, para algunos clientes de las compañías de seguros, la exclusión puede parecer arbitraria. Incluso cuando la exclusión se basa en el principio de quien llegó primero, para muchos parece estar relacionada con una relación exclusiva entre los aseguradores y las grandes empresas forestales. En cierto modo, el acceso geográficamente desigual a los seguros en Chile representa una externalidad negativa de la práctica de la gestión del riesgo basada en los seguros y puede interpretarse como el resultado de la descontextualización del riesgo y del conocimiento del riesgo.

\section{La descontextualización del riesgo y del conocimiento del riesgo}

Los expertos en riesgos tienden a analizar y manejar diferentes riesgos en forma aislada (MacKenzie 2011). De manera similar, los actores expuestos al riesgo comprenden epistemológicamente las causas y efectos de los riesgos como si estuvieran separados unos de otros (Rosa et al. 2014). Por lo tanto, conceptualmente, tiene mucho sentido separar la dimensión física de las pérdidas potenciales de la dimensión discursiva de cómo las personas perciben, comunican y construyen socialmente el riesgo originalmente. En el curso de estas prácticas, el conocimiento sobre los riesgos es fácilmente descontextualizado, también en un sentido espacial (November, 2008).

Las empresas forestales y de seguros en Chile diseñan y trabajan con modelos económicos para la toma de decisiones que se consideran (y han probado ser) útiles para el manejo del riesgo. Al hacerlo, claramente descontextualizan el riesgo de incendios forestales en un sentido espacial. Las empresas forestales, por ejemplo, descontextualizan el riesgo y el conocimiento del riesgo en el curso de sus estrategias de homogeneización y estandarización en el manejo de las áreas forestadas. Homogeneizan el espacio mediante la compra de áreas adyacentes, que luego utilizan para la reforestación, y estandarizan el espacio mediante el establecimiento de las mismas rutinas de gestión en sus plantaciones. Las rutinas no solo estandarizan el conocimiento del riesgo, sino que también desacoplan el riesgo de la unidad de espacio, en el curso de la cual los bosques de plantación se transforman en activos que pueden valorarse de manera uniforme y de acuerdo con las economías de escala. Un área forestal manejada como monocultivo descontextualiza las condiciones específicas del sitio. Ignora el hecho de que, desde el punto de vista del riesgo, algunos sitios podrían haber sido más adecuados para otras especies arbóreas.

La descontextualización de los conocimientos relacionados con los riesgos de incendios forestales también puede verse en el hecho de que las grandes empresas forestales incluso consiguen adquirir seguros para sus plantaciones en comunidades políticamente inestables, es decir, comunidades en las que las compañías de seguros están planificando una estrategia de racionamiento para el mercado. Las grandes empresas forestales generalmente poseen y manejan áreas forestales distribuidas en varias regiones forestales chilenas, y debido a que estas son homoge- 
neizadas y manejadas de manera estandarizada y comparable, pueden adquirir un paquete de pólizas de seguros de las compañías de seguros. Los riesgos de incendios forestales en las zonas de alto riesgo se compensan con los riesgos en las zonas menos amenazadas. El conocimiento exacto de las circunstancias del riesgo en el paquete se vuelve difuso y descontextualizado.

Al comprar un paquete de seguros con propiedad forestal dispersa espacialmente, también la aseguradora descontextualiza el riesgo y su conocimiento del riesgo, lo que inicialmente les da ventajas: un paquete de seguros no solo garantiza la aplicación de la ley de los grandes números, sino que también ofrece a la aseguradora la oportunidad de repartir los riesgos en el espacio, lo que reduce su riesgo de fracaso total. Su riesgo total ya no está determinado por las condiciones de un lugar determinado. Sin embargo, la distribución espacial del riesgo también reduce su interés en estudiar el conocimiento específico del contexto sobre las causas y efectos exactos de los incendios forestales individuales. Además, la cantidad de deducibles que los asegurados aceptan en los contratos de seguro reduce aún más los incentivos de la aseguradora para adquirir un conocimiento detallado de los riesgos relacionados con el contexto. Por último, la descontextualización del riesgo aumenta su cuantificabilidad, lo que permite su traducción al lenguaje de los mercados financieros, y por ende, al asegurador revender parte del riesgo a las compañías de reaseguro. La práctica generalizada de descontextualizar el riesgo y el conocimiento sobre los riesgos de incendios forestales en Chile, junto con la reducción de los incentivos para generar nuevos conocimientos, por ejemplo, sobre las causas y los efectos de los incendios forestales, puede ser un obstáculo en la gobernanza del riesgo según la sociedad.

\section{Conclusión}

Los riesgos de incendios forestales en Chile tienen diferentes orígenes y consecuencias espacio-temporales. Resultan ser epistemológicamente complejos, ambiguos e inciertos dependiendo del contexto socio-económico y socio-político de su aparición. Las diferentes causas y efectos físicos y antropológicos de los incendios no pueden atribuirse claramente (= complejidad). Las causas inexplicables dan lugar a varias explicaciones lógicas, que se contradicen entre sí (=ambigüedad). En muchos casos, también hay una falta de sensibilidad al propio desconocimiento sobre el riesgo (=incertidumbre). En suma, el conocimiento sobre las causas y efectos de los incendios forestales en Chile es heterogéneo y geográficamente disperso entre los actores sociales. Es evidente que los riesgos de incendios forestales están construidos socialmente. Dependen de cómo se interpreten los hallazgos de las ciencias naturales y sociales como si estuvieran estrechamente entrelazados.

Centrándose en las explicaciones económico-geográficas, este estudio no solo dejó claro que los incendios forestales son un riesgo profundamente geográfico, sino que también representan un desafío fundamentalmente colectivo para las regiones forestales chilenas. Un solo incendio, independientemente de su origen, puede afectar y destruir la propiedad forestal de los actores vecinos. En consecuencia, era de esperar que se establecieran relaciones de vecindad, es decir, enfoques cooperativos y colectivos en la gestión de riesgos. Sin embargo, resultó que las empresas forestales prefieren organizar la gestión de riesgos de manera individual. Paralelamente, compran pólizas de seguros para transferir parte del riesgo a las compañías de seguros. Organizan 
esta interacción bilateralmente basándose en el diseño del contrato y en los precios de mercado negociados para el riesgo.

Las empresas forestales y las compañías de seguros en Chile parecen haber encontrado una forma complementaria de manejar el riesgo compartiendo el conocimiento técnico creado conjuntamente como parte de sus prácticas rutinarias de manejo del riesgo. Ambos eligen un enfoque de manejo que reduce la complejidad, desacoplando el riesgo y descontextualizando el conocimiento del riesgo para hacer que los riesgos de incendios forestales sean calculables y manejables de manera mutuamente beneficiosa. Convienen, por ejemplo, en los mecanismos de precios para los contratos de seguros como lenguaje estandarizado, lo que tiene como consecuencia que el conocimiento geográfico detallado sobre los riesgos de incendios forestales en Chile sea explícitamente descontextualizado y reducido. También siguen estrategias complementarias de evitación de riesgos cuando los riesgos se caracterizan por ser ambiguos o inciertos.

Los catastróficos incendios forestales de 2017 revelaron los límites de las prácticas estandarizadas de gestión de riesgos. La ley de los grandes números y, por lo tanto, la calculabilidad del riesgo, en términos generales, se vuelve menos efectiva cuando ocurren grandes e improbables eventos o cuando las causas de los riesgos son sistémicamente interdependientes. Este último es el peor caso para el mercado de los seguros. Las relaciones sistémicas pueden esconderse detrás de características de ambigüedad o incertidumbre. Además, existe el gran peligro de que si los actores sólo siguen sus propios discursos dominantes sobre el riesgo e ignoran las observaciones e interpretaciones que se deben a las diferentes condiciones del contexto espacio-temporal del riesgo, los cambios dinámicos en las relaciones entre los factores ambientales y antropogénicos de los incendios forestales en Chile sigan siendo invisibles para los tomadores de decisiones sobre el riesgo. La enorme extensión de los incendios forestales de 2017 puede atribuirse sin duda en primer lugar a las condiciones climáticas extremas. Pero también hay numerosos indicios de que los incendios han sido provocados intencionalmente en un amplio frente. Desafortunadamente, sus antecedentes exactos siguen siendo desconocidos hasta el día de hoy (Saavedra, 2017).

Esto requiere un esfuerzo coordinado de gestión de riesgos que dé una ventaja explícita al conocimiento regional y que esté diseñado como un proceso de aprendizaje a largo plazo. La gestión de riesgos cubre principalmente las visiones individuales del riesgo. En contraste, la gobernanza del riesgo ofrece un enfoque holístico que asigna las responsabilidades sociales de los tomadores de riesgo y también considera las externalidades negativas (en su variación espacio-temporal) para otros actores de la sociedad. Por supuesto, esto no significa que la gestión de riesgos no tenga ningún valor. El concepto de gobernanza de riesgos del Consejo Internacional de Gobernanza de Riesgos, un grupo de reflexión internacional que tiene por objeto mejorar la comprensión y la evaluación de los riesgos y de las ambigüedades que entrañan, por ejemplo, integra la gestión de riesgos en un proceso más amplio de gobernanza de riesgos. La comunicación de riesgos, que incluye la transmisión de datos sobre riesgos, pero también la transferencia de conocimientos avanzados sobre riesgos, constituye el vínculo de conexión en el proceso de gobernanza, que es circular y reflexivo por naturaleza (Renn, 2008: 374). La gobernanza del riesgo tiene en cuenta los atributos de ambigüedad e incertidumbre en el conocimiento y exige la apertura de debates sobre el riesgo y la participación de muchas partes interesadas de la sociedad en los procesos de evaluación y valorlzacón del riesgo. En particular, se trata de la transparencia, que permite el aprendizaje colectivo para una sociedad de riesgo más resistente. 
La complejidad, ambigüedad e incertidumbre en el conocimiento de los riesgos de incendios forestales en Chile deben tomarse en serio. Estos atributos se originan desde la perspectiva de las ciencias sociales sobre el riesgo. Desde el punto de vista empírico, no siempre es posible separarlas unas de otras, por lo que no pueden tener como objetivo estructurar de manera uniforme la gobernanza del riesgo. Sin embargo, su uso transparente en la gestión del riesgo o en la gobernanza del riesgo tiene consecuencias. Comunica a la sociedad cómo se define y legitima el riesgo en el futuro (véase Bustos et al, 2019 para un argumento similar relacionado con la gestión regional de crisis en Chile). La falta de participación de la sociedad y de aceptación de los entornos normativos puede provocar reacciones adversas y resistencia (véase, por ejemplo, el estudio de caso sobre incendios forestales en California de Simon \& Dooling, 2013). Dado que los incendios forestales son inmanentemente espaciales, la escala regional promete ser adecuada para iniciar la organización de los procesos relacionados con la gobernanza del riesgo. A nivel regional, las interrelaciones cambiantes en la formación de riesgos pueden observarse e interpretarse desde el principio (Müller-Mahn et al, 2018). Sin embargo, como han demostrado el artículo, los riesgos de incendios forestales pueden separarse técnicamente de la dimensión espacial, especialmente en términos de sus efectos económicos. La descontextualización es lo que permite hacer frente al riesgo desde el punto de vista financiero.

La relación complementaria entre formas jerárquicas y mercantiles de la gestión de riesgos es recíproca, también para los enfoques de gobernanza de riesgos. Algunos de los conocimientos y orientaciones sobre determinadas prácticas de gestión de riesgos a escala regional proceden directamente del sector de los seguros. A través del diseño de contratos y negociaciones, las aseguradoras ofrecen incentivos directos e indirectos para desacoplar el riesgo y aprender a gestionarlo de forma rutinaria. El grado en que las diferentes formas de gestión del riesgo se complementan entre sí en un contexto regional de gobernanza y conducen a un mayor nivel de conocimiento local-global sigue siendo un tema pendiente que requiere esfuerzos de investigación adicionales más allá del ejemplo de los incendios forestales en Chile.

\section{Referencias bibliográficas}

AGROSEGUROS. Seguro de explotaciones forestales - condiciones (=Linea 319/Plan 2018). Santiago de Chile, 2018.

ALTAMIRANO, A.; SALAS, C.; YAITUL, V.; SMITH-RAMIREZ, C. \& ÁVILA, A. Influencia de la heterogeneidad del paisaje en la ocurrencia de incendios forestales en Chile Central. Revista de Geografía Norte Grande, 2013, N 55, p. 157-170.

ARNALDOS, J. \& NAVALÓN, X. Manual de ingeniería básica para la prevención y extinción de incendios forestales. Madrid: Ediciones Mundi-Prensa, 2004.

ASPERS, P. Knowledge and valuation in markets. Theory \& Society, 2009, № 38, p. 111-131.

AYLWIN, J. Incendios y modelo forestal, una estrecha relación (publicado en el periódico www. theclinic.cl, 07.02.2017). 
BARTON, J. \& ROMÁN, Á. Social movement strategies for articulating claims for socio-ecological justice: local asymmetries in the Chilean forestry sector. Globalizations, 2012, № 9, p. 869-885.

BECK, U. La sociedad del riesgo: hacia una nueva modernidad. Madrid: Paidos, 1998.

BECK, U. Living in the world risk society. Economy and Society, 2006, N 35, p. 329-345.

BECKER, M. \& KNUDSEN, T. The role of routines in reducing pervasive uncertainty. Journal of Business Research, 2005, N 58, p. 746-757.

BECKERT, J. Fictional expectations and capitalist dynamics. Cambridge: Harvard University Press, 2016.

BOTTARO, G.; ROCO, L.; PETTENELLA, D.; MICHELETTI, S. \& VANHULST, J. Forest plantations' externalities: An application of the analytic hierarchy process to non-industrial forest owners in Central Chile. Forests, 2018, Nº 9, p. 1-21.

BRUGNACH, M. \& INGRAM, H. Ambiguity: the challenge of knowing and deciding together. Environmental Science \& Policy, 2012, N 15, 60-71.

BUSTOS, B.; LUKAS, M.; STAMM, C. \& TORRE, A. Neoliberalismo y gobernanza territorial: propuestas y reflexiones a partir del caso de desde Chile. Revista de Geografía Norte Grande, 2019, № 73 , p. 161-183.

CABANTOUS, L. Ambiguity aversion in the field of insurance: Insurers' attitude to imprecise and conflicting probability estimates. Theory and Decision, 2007, N 62, p. 219-240.

CASTILLO, M. E.; MOLINA-MARTÍNEZ, J. R.; RODRÍGUEZ, F. \& JULIO, G. H. A territorial fire vulnerability model for Mediterranean ecosystems in South America. Ecological Informatics, 2013, № 13, p. 106-113.

CAVIEDES, J. A. Construyendo sobre cenizas. ¿Son utilizados los incendios forestales como una herramienta informal para la expansión urbana de Chile central? (=Tesis para obtener el grado académico de Magister en Asentamientos Humanos y Medio Ambiente, Pontificia Universidad Católica de Chile). Santiago de Chile, 2017.

CHICHILNISKY, G. \& HEAL, G. Managing unknown risks. The future of global reinsurance. Portfolio Management, 1998, $N^{\circ} 24$, p. 85-91.

CLAPP, A. R. Creating Competitive Advantage: Forest Policy as Industrial Policy in Chile. Economic Geography, 1995, № 71, p. 273-296.

CMPC [Compañía Manufacturera de Papeles y Cartones] Integrated Report CMPC 2017. Santiago de Chile, 2018. 
CONAF [Corporación Nacional Forestal] Descripción y efectos 'tormenta de fuego', 18 de enero al 5 de febrero de 2017, regiones O'Higgins, el Maule y Biobío. Santiago de Chile, 2017.

CONAF [Corporación Nacional Forestal] Ley sobre recuperación del bosque nativo y fomento forestal y reglamentos. Santiago de Chile, 2008.

CONAF [Corporación Nacional Forestal]. Estadísticas publicadas en la página del web http://www. conaf.cl/centro-de-documentacion/, 2018.

CROUCH, C. \& KEUNE, M. The governance of economic uncertainty: beyond the 'new social risks' analysis. En: Bonoli, G. \& Natali, D. The Politics of the New Welfare State. Oxford: Oxford University Press, 2012, p. 45-67.

DEAN, B.; DOYLE, A. \& ERICSON, D. Insurance as governance. Toronto: University of Toronto Press, 2003.

DÍAZ-HORMAZÁBAL, I. \& GONZÁLEZ, M. E. Análisis espacio-temporal de incendios forestales en la región del Maule, Chile. Bosque, 2016, №37, p. 147-158.

DRANSCH, D.; ROTZOLL, H. \& POSER, H. The contribution of maps to the challenges of risk communication to the public. International Journal of Digital Earth, 2010, N³, 292-311.

ERICSON, R.V. \& DOYLE, A. Uncertain business. Risk, insurance, and the limits of knowledge. Torono: University of Toronto Press, 2004.

FARRÉ, J. Comunicación de riesgo y espirales del miedo. Nueva Epoca, 2005, № 3, p. 95-119.

FOCACCI, G. Investigan relación de incendios forestales con plaga de avispas y pago de seguros (publicado en el periódico https://www.biobiochile.cl, 04.07.2017).

FREEMAN, P. \& KUNREUTHER, H. Managing environmental risk through insurance. Dordrecht: Kluwer, 1997.

GATICA, F. Prácticas empresariales con potencial de vinculación territorial: el caso del sector forestal chileno. Revista de Geografía Norte Grande, 2012, N 52, p. 127-143.

GERBER, J.-F. Conflicts over industrial tree plantations in the South: who, how and why? Global Environmental Change, 2011, № 21, p. 165-176.

GLÜCKLER, J.; REHNER, J. \& HANDKE, M. Governance, Networks and Territory: Editorial. Revista de Geografía Norte Grande, 2019, xxx.

Gobierno de Chile (2017): Chile y la tormenta de fuego (=Informe Incendios Forestales - Enero-Febrero 2017). Santiago de Chile: Ministerio del Interior y Seguridad Pública. 
GÓMEZ-GONZÁLEZ, S.; GONZÁLEZ, M. E.; PAULA, S.; DÍAZ-HORMAZÁBAL, I.; LARA, A. \& DELGADO-BAQUERIZO, M. Temperature and agriculture are largely associated with fire activity in Central Chile across different temporal periods. Forest Ecology and Management, 2019, $\mathrm{N}^{\circ} 433$, p. $535-$ 543.

GONZÁLEZ, M.; GÓMEZ-GONZÁLEZ, S.; LARA, A.; GARREAUD, R. \& DÍAZ-HORMAZÁBAL, I. The 2010-2015 megadrought and its influence on the fire regime in central and south-central Chile. Ecosphere, 2018, № 9, Article e02300.

GONZÁLEZ-HIDALGO, M. \& ZOGRAFOS, C. How sovereignty claims and negative emotions influence the process of subject-making: Evidence from a case of conflict over tree plantations from Southern Chile. Geoforum, 2017, Nº 78, p. 61-73.

HACKING, I. The taming of chance. Cambridge: Cambridge University Press, 1990.

HANSON, J. D. \& KYSAR, D. A. Taking behavioralism seriously: The Problem of market manipulation. New York University Law Review, 1999, № 74, p. 632-749.

HAYEK, F. The use of knowledge in society. The American Economic Review, 1945, № 35, p. 158169.

HELLWIG, M. Moral hazard and monopolistically competitive insurance markets. The Geneva Papers on Risk and Insurance - Issues and Practice, 1983, N 8, p. 44-71.

ILIN, T. \& VARGA, L. The uncertainty of systemic risk. Risk Management, 2015, № 17, p. 240-275.

INFOR [Instituto Forestal] Estadísticas publicadas en la página del web http:// https://wef.infor. $\mathrm{cl} /, 2018$.

JARZABKOWSKI, P.; BEDNAREK, R. \& SPEE, P. Making markets for acts of god. Oxford: Oxford University Press, 2015.

JOHANSEN, I.; RAUSAND, M. Ambiguity in risk assessment. Safety Science, 2015, Nº 80, p. 243-251.

JULIO, G. Tendencias del problema de los incendios forestales en Chile. Revista Mundo Forestal, 2014, N²6, No. 1, p. 11-16.

KALTHOFF, H. Practices of Calculation: Economic Representations and Risk Management. Theory, Culture \& Society, 2005, N²2, p. 69-97.

KESSLER, O. What price culture? Calculation, commensuration, contingency, and authority in financial practices. En: JESSOP, B.; YOUNG, B. \& SCHERRER, C. Financial cultures and crisis dynamics. Abingdon: Routledge, 2015, p. 85-105.

KNIGHT, F. Risk, uncertainty and profit. Boston: Houghton, 1921. 
LATORRE, J. I. \& ROJAS, N. El conflicto forestal en territorio mapuche hoy. Ecología Política, 2016, $N^{\circ} 51$, p. 84-87.

LITTLE, C.; LARA, A.; MCPHEE, J. \& URRUTIA, R. Revealing the impact of forest exotic plantations on water yield in large-scale watersheds in South-Central Chile. Journal of Hydrology, 2009, $\mathrm{N}^{\circ}$ 374, p. 162-170.

LOEWE, V.; CORTI, D.; RUIZ, J. M. \& LOBO, F. La evaluación del riesgo de incendio en plantaciones forestales para mejorar el acceso de la pyme a seguros contra incendios (=Paper presented at the 7th Congreso Forestal Español, 26.-30.06.2017, Cáceres, Extremadura).

LUHMANN, N. Sociología del riesgo. México: Universidad Latinoamericana, 2006.

LUNDQVIST, S.A. Why firms implement risk governance - Stepping beyond traditional risk management to enterprise risk management. Journal of Accounting and Public Policy, 2015, $\mathrm{N}^{\circ}$ 34, p. 441-466.

MACKENZIE, D. The Credit Crisis as a Problem in the Sociology of Knowledge. American Journal of Sociology, 2011, Nº 116, p. 1778-1841.

MAPUCHEEXPRESS. Revuelo caso incendios e intencionalidad: zonas devastadas estaban infestadas por plagas en plantaciones forestales (publicado en el periódico http://www.mapuexpress. org, 04.02.2017).

MARTIN, W. E.; MARTIN, I. M. \& KENT, B. The role of risk perceptions in the risk mitigation process: The case of wildfire in high-risk communities. Journal of Environmental Management, 2009, $\mathrm{N}^{\circ} 91$, p. 489-498.

MARTÍNEZ-FERNÁNDEZ, J.; MARTÍNEZ-VEGA, J. \& MARTÍN, P. El factor humano en los incendios forestales: Análisis de factores socio-económicos relacionados con la incidencia de incendios forestales en España. En: Chuvieco, E. \& Martín, P. Nuevas tecnologías para la estimación del riesgo de incendios forestales. Madrid: CSIC, Instituto de Economía y Geografía, 2004, p. 101-142.

MCWETHY, D. B.; PAUCHARD, A.; GARCÍA, R. A.; HOLZ, A.; GONZÁLEZ, M. E.; VEBLEN, T. T.; STAHL, J. \& CURREY, B. Landscape drivers of recent fire activity (2001-2017) in south-central Chile. PLOS ONE, 2018, No 13, e0201195.

MERMOZ, M.; KITZBERGER, T. \& VEBLEN, T.T. Landscape influences on occurrence and spread of wildfires in Patagonian forests and shrublands. Ecology, 2005, Nº 86, p. 2705-2715.

MILLONES, M. ¿Quiénes incendiaron Chile? (publicado en el periódico https://www.elmostrador. cl, 03.08.2017).

MONTALBA-NAVARRO, R. \& CARRASCO, N. Modelo forestal chileno y conflicto indígena ¿ecologismo cultural mapuche? Ecología Política, 2003, N²6, p. 63-78. 
MORENO DEL VALLE, F. Orientaciones y contradicciones en la Ley de Bosque Nativo. Justicia Ambiental, 2015, Nº 7, p. 164-185.

MÜLLER-MAHN, D.; EVERTS, J. \& STEPHAN, C. Riskscapes revisited - Exploring the relationship between risk, space and practice. Erdkunde, 2018, N 72, p. 197-213.

MUNDO FORESTAL Debate incendios forestales entre Susana Gómez-González y Roberto Cornejo Espósito. Revista Mundo Forestal, 2017, №32, p. 16-29.

NOVEMBER, V. Commentary on the spatiality of risk. Environment and Planning A, 2008, $\mathrm{N}^{\circ} 40, \mathrm{p}$. 1523-1527.

O'FLANAGAN, P. ¿Incendiarismo o ecocidio en la Iberia Atlántica? La transformación del paisaje a causa del fuego: reflejo de los problemas socioeconómicos. Polígonos, 1997, № 7, p. 77-96.

O'Malley, P. Risk, Uncertainty and Government. London: Glasshouse Press, 2004.

PERROW, C. Complex Organizations. A Critical Essay. New York: McGraw-Hill, 1986.

RENN, O. Risk Governance. Coping with Uncertainty in a Complex World. London: Earthscan, 2008.

REYES, R. \& NELSON, H. A tale of two forests. Why forests and forest conflicts are both growing in Chile. International Forestry Review, 2014, No 16, p. 379-388.

RICHTER, R. \& FURUBOTN, E. G. Institutions and Economic Theory; The Contribution of the New Institutional Economics. Chicago: Ann Arbor, 1997.

ROJAS, N. \& MIRANDA, O. Dinámica sociopolítica del conflicto y la violencia en territorio mapuche. Particularidades históricas de un nuevo ciclo en las relaciones contenciosas. Revista de Sociología, 2015, N³0, p. 33-69.

ROSA, E.A.; RENN, O. \& MCCRIGHT, A.M. The risk society revisited. Social theory and governance. Philadelphia: Temple University Press, 2014.

SAAVEDRA, O. Productores de madera: "Tenemos temores fundados... Nos enfrentamos al terrorismo" (publicado en el periódico http://www.economiaynegocios.cl, 29.01.2017).

SALVATI, L. \& RANALLI, F. Land of fires: Urban growth, economic crisis, and forest fires in Attica, Greece. Geographical Research, 2015, N 53, No. 1, 68-80.

SEGOVIA, P.; BASULTO, O. \& ZAMBRANO, P. Imaginarios sociales y representaciones: su aplicación a análisis discursivos en tres ámbitos diferentes. EMPIRIA. Revista de Metodología de Ciencias Sociales, 2018, N 41, p. 79-102.

SEGUEL, A. Chile: mega incendios forestales, crímenes empresariales e impunidad (publicado en el Boletín 238 del Movimiento Mundial por los Bosques Tropicales, https://wrm.org.uy, 09.07.2018). 
SEPÚLVEDA, J. La historia de los autoatentados incendiarios de Forestal Mininco en 1999 (publicado en el periódico http://www.gamba.cl, 04.01.2013).

SIMON, G. L. \& DOOLING, S. Flame and fortune in California: The material and political dimensions of vulnerability. Global Environmental Change, 2013, Nº 23, p. 1410-1423.

SOIN, K. \& COLLIER, P. Risk and risk management in management accounting and control. Management Accounting Research, 2013, N² 24, p. 82-87.

STIGLITZ, J. E. Risk, incentives and insurance: The pure theory of moral hazard. The Geneva Papers on Risk and Insurance, 1983, Nº 8, p. 4-33.

TAPIA, G. \& CASTILLO, M. Propuesta de diseño de un sistema de torres de detección de incendios forestales: aplicación a la región Metropolitana de Chile central. Bosque, 2014, №35, p. 399-412.

ÚBEDA, X. \& SARRICOLEA, P. Wildfires in Chile: A review. Global and Planetary Change, 2016, Nº 146, p. 152-161.

VAN ASSELT, M. \& RENN, O. Risk governance. Journal of Risk Research, 2011, No 14, p. 431-449.

VAN DAM, C. Empresas forestales y comunidades rurales en el centro-sur de Chile: externalidades sociales de un modelo ,exitoso'. Debate Agrario, 2006, № 40/41, p. 225-243.

VAN DIJK, T. Discourse and Knowledge. Cambridge: Cambridge University Press, 2014.

VAN HOLT, T.; BINFORD, M. W.; PORTIER, K. M.; VERGARA, R. A stand of trees does not a forest make: Tree plantations and forest transitions. Land Use Policy, 2016, N 56, p. 147-157.

VELEZ, R. La defensa contra incendios forestales. Fundamentos y experiencias. Madrid: McGraw-Hill, 2009.

WILLIAMSON, O. E. Calculativeness, trust and economic organization. Journal of Law and Economics, 1993, No 36, p. 453-486. 
